\title{
AN ABSTRACT RADON-NIKODYM THEOREM
}

\author{
BY \\ C. E. RICKART
}

1. Introduction. Let $\mathcal{F}$ be a $\sigma$-field of subsets of an abstract set $M$ and let $m(e)$ be a non-negative measure function defined on $\mathcal{F}$. The classical RadonNikodym theorem $\left[17\right.$, p. 36]( $\left.{ }^{1}\right)$ states that, if $M$ is the union of a countable number of sets of finite measure, then a necessary and sufficient condition for a completely additive real function $R(e)$, defined over $\mathcal{F}$, to be a Lebesgue integral (with respect to $m(e)$ ) is that $R(e)$ be absolutely continuous relative to $m(e)$. Our purpose is to extend this theorem to functions with values in an arbitrary Banach space and apply the resulting theorem to obtain an integral representation for the general bounded linear transformation on the space of summable functions to an arbitrary Banach space. A number of writers $[4,6$, $7,8,11,12,13,14]$ have obtained similar extensions; however they have all imposed restrictions either on the Banach space or on the completely additive functions considered. The theorem proved here is free of all such restrictions. It is evident that any such generalization of the Radon-Nikodym theorem will involve a corresponding generalization of the Lebesgue integral, of which there are many. A variation of an integral studied in detail by B. J. Pettis $\left({ }^{2}\right)$ will be used here.

A point function $x(p)$ defined on $M$ to a Banach space $\mathfrak{X}$ is said to be Pettis integrable [12] provided there exists a function $X(e)$ on $\mathcal{F}$ to $\mathfrak{X}$ such that, for each element $\bar{x}$ of the space $\overline{\mathfrak{X}}$ adjoint to $\mathfrak{X}$ and each element $e$ of $\mathcal{F}$, the function $\bar{x}(x(p))$ is Lebesgue integrable on the set $e$ to the value $\bar{x}(X(e))$. Whenever $X(e)$ exists, it is completely additive and absolutely continuous relative to $m(e)$. On the other hand, Pettis $[12$, p. 303] gave an example of a completely additive function which is absolutely continuous but is not an integral in his sense. This shows that the ordinary Pettis integral cannot appear in a general Radon-Nikodym theorem. However, without changing essentially the definition or general properties of the integral, we can enlarge the class of functions admissable for integration (so that it contains certain functions other than point functions) and thus obtain an integral which will serve our purposes.

The class of functions which we will admit for integration consists of all multivalued set functions $x(e)$ defined for elements of $\mathcal{F}$ having finite, nonzero

Except for $\$ 5$, the contents of this paper were presented to the Society, September 12, 1943. The results in $\$ 5$ were presented February 27,1944 , under the title Representation of linear transformations on summable functions. Received by the editors December 30, 1943.

(1) Numbers in brackets refer to the Bibliography at the end of the paper.

(2) See also Dunford [5, p. 338]. 
measure and subject to the condition that $e^{\prime} \subseteq e$ imply $x\left(e^{\prime}\right) \subseteq x(e)$. Such functions are termed contractive. To every point function $x(p)$ there corresponds the contractive function $x(e)$, whose values on $e$ are the values taken on by $x(p)$ as $p$ ranges over $e$. It is well known that a trivial reformulation of one definition of the Lebesgue integral (and hence the Pettis integral) enables one to consider integration of contractive functions. Moreover, all properties of the integral not depending directly on point functions remain unchanged, and integration of a contractive function, which is associated in the above manner with a point function, is equivalent to ordinary integration of the point function. Since we no longer have to do with point functions, it is possible to replace the restriction that $\mathcal{F}$ be a $\sigma$-field of sets of points by the weaker assumption that $\mathcal{F}$ be a countably additive Boolean algebra.

The introduction of functions other than point functions admittedly reduces the force of the Radon-Nikodym theorem. On the other hand, if one were to insist on point functions, it would be necessary to define an integral more general than the Pettis integral. However, it is easy to prove $[12$, p. 281] that any integration process, which gives the Lebesgue integral in the real case and which is preserved under linear continuous transformation of the Banach space into a second Banach space, is contained in the Pettis integral. Therefore, although it may be possible to obtain the general Radon-Nikodym theorem involving only point functions, the integral used would necessarily lack some desirable elementary properties.

A few notations and definitions are collected in $\$ 2 . \$ 3$ contains a defunition of the variation of the Pettis integral suggested above along with some of its properties. The Radon-Nikodym theorem is proved in $\$ 4$ and is applied in $\$ 5$ to obtain an integral representation for the general, bounded, linear transformation on the space $L(M)$ of summable functions to an arbitrary Banach space.

2. Notations and definitions. Throughout this and the following two sections, $\mathcal{F}$ will denote a countably additive Boolean algebra. In lattice terminol: ogy, $\mathcal{F}$ is a $\sigma$-complete, complemented, distributive lattice $[2$, p. 88]. The lattice operations of join, meet, and complementation will be indicated respectively by $e \cup e^{\prime}, e \cap e^{\prime}$, and $C e$. The join and meet of a countable number of elements $e_{n}$ will be written $\sum e_{n}, \prod e_{n}$ respectively. Since $\mathcal{F}$ is $\sigma$-complete, these will always exist in $\mathcal{F}$. The extreme elements of $\mathcal{F}$ will be denoted by 0 (null or least element) and $M$ (unit or greatest element). An element $e$ is said to be contained in a second element $e^{\prime}$ provided $e \cap e^{\prime}=e$. This inclusion will be written $e \subseteq e^{\prime}$ or $e^{\prime} \supseteq e$. Two elements $e, e^{\prime}$ are disjoint provided $e \cap e^{\prime}=0$. An ordinary $\sigma$-fold of sets is a countably additive Boolean algebra in which join, meet, and complement are the corresponding set-theoretic notions.

A disjoint sequence $\left\{e_{i}\right\}$ of elements of $\mathcal{F}$ (that is, $e_{i} \cap e_{j}=0$, for $i \neq j$ ) such that $\sum e_{i}=e$ is called a subdivision of $e$. The product of two subdivisions of $e, \Delta=\left\{e_{i}\right\}$ and $\Delta^{\prime}=\left\{e_{j}^{\prime}\right\}$, is defined to be the sequence $\Delta \Delta^{\prime}=\left\{e_{i} \cap e_{j}^{\prime}\right\}$. 
From the distributive properties of $\mathcal{F}$, it follows easily that this product is also a subdivision of $e$. It also follows from the distributivity that every subdivision $\left\{e_{i}\right\}$ of $M$ induces a subdivision $\left\{e \cap e_{i}\right\}$ of the element $e$. One subdivision, $\Delta^{\prime}=\left\{e_{i}^{\prime}\right\}$, is said to be finer than a second, $\Delta=\left\{e_{j}\right\}$, provided every element $e_{i}^{\prime}$ is contained in some $e_{j}$. We write $\Delta^{\prime} \geqq \Delta$.

The class of elements $x$ which satisfy a given property $P$ will be denoted by $\{x \mid P\}$. The small Greek letter $\pi$ will always stand for a finite set of positive integers, and $\sum_{\pi}$ (for example) will mean summation over all indices contained in $\pi$.

Let $\mathfrak{X}$ be an arbitrary linear set $[1$, p. 26] with real multipliers and zero element $\theta$. If $X, X^{\prime}$ are any two subsets of $\mathfrak{X}$, then $X+X^{\prime}$ is defined to be the set $\left\{x+x^{\prime} \mid x \in X, x^{\prime} \in X^{\prime}\right\}$. Similarly, if $X \subseteq \mathfrak{X}$ and $A$ is a set of real numbers, then $A X$ is the set $\{r x \mid r \in A, x \in X\}$; in particular, if $r$ is a single real number, $r X=\{r x \mid x \in X\}$. We will assume $\mathfrak{X}$ to be a linear topological space of the type introduced by J. von Neumann [10, p. 4]. The system of neighborhoods of $\theta$, which defines the topology, will be denoted by $U$. Individual elements of $U$ are denoted by $V . \mathfrak{X}$ is said to be convex provided, for every $V, V+V \subseteq 2 V$. Under this condition, the closure of each of the neighborhoods is a convex set in the ordinary sense $\left(^{3}\right)$. Another important property of the neighborhoods, in case $\mathfrak{X}$ is convex, is that $0<r<r^{\prime}$ implies $r V \subseteq r^{\prime} V$. We will be primarily interested in the case in which $\mathfrak{X}$ is a Banach space under one of its weak neighborhood topologies. A weak neighborhood system $V_{r}$ consists of all neighborhoods of the form

$$
V=\left\{x|| \bar{x}_{i}(x) \mid<\epsilon ; \epsilon>0, \bar{x}_{i} \in \Gamma, i=1,2, \cdots, n\right\},
$$

where $\Gamma$ is a subset of the space $\overline{\mathfrak{X}}$ adjoint to $\mathfrak{X}$. In case $\Gamma$ is a total $\left({ }^{4}\right)$ subset of $\bar{X}$, the system $V_{\Gamma}$ determines in $\mathfrak{X}$ a convex $\left(^{(5)}\right.$ topology of the von Neumann type. Whenever a Banach space is under consideration, $U$ will always be understood to mean the system $V_{\Gamma}$, when $\Gamma=\overline{\mathfrak{X}}$.

Two subsets $X, X^{\prime}$ of $\mathfrak{X}$ are said to be equal within $V$ provided $X \subseteq X^{\prime}+V$ and $X^{\prime} \subseteq X+V$. Two sequences $\left\{X_{n}\right\},\left\{X_{n}^{\prime}\right\}$ of subsets of $\mathfrak{X}$ are said to be summably equal within $V$ provided there exists $\pi_{V}$ such that $\pi \supseteq \pi_{V}$ implies $\sum_{\pi} X_{n}, \sum_{\pi} X_{n}^{\prime}$ are equal within $V$. A sequence $\left\{X_{n}\right\}$ of subsets of $\mathfrak{X}$ is said to be unconditionally summable $[13$, p. 118$]$ to the element $x \in \mathfrak{X}$ with respect to $V$ provided there exists $\pi_{V}$ such that

$$
\pm\left\{x-\sum_{\pi} X_{n}\right\} \subset V, \quad \pi \supseteq \pi_{V}
$$

If each set $X_{n}$ contains only one element $x_{n}$, then the condition that $\left\{X_{n}\right\}$

$\left(^{3}\right)$ A set $X \subseteq X$ is convex provided $x_{i} \in X, r_{i} \geqq 0$ and $\sum r_{i}=1$ imply $\sum r_{i} x_{i} \in X(i=1,2, \cdots, n)$.

(4) A subset $\Gamma \subseteq \bar{x}$ is said to be total $[1$, p. 42] provided $\bar{x}(x)=0$, for every $\tilde{x} \in \Gamma$, implies $x=\theta$.

(s) As a matter of fact, each of the neighborhoods $V$ is itself a convex set. Also $-V=V$. 
be unconditionally summable to $x$ with respect to every $V \in U$ is simply the condition that the series $\sum x_{n}$ be unconditionally convergent to the value $x$ [9].

A single-valued function $X(e)$ defined on a subset of $\mathcal{F}$ to $\mathfrak{X}$ is said to be completely additive relative to $U$ provided the series $\sum X\left(e_{n}\right)$ converges unconditionally to $X\left(\sum e_{n}\right)$ in the topology $U$, whenever $\left\{e_{n}\right\}$ is a countable sequence of mutually disjoint elements such that $X\left(\sum e_{n}\right)$ and $X\left(e_{n}\right)$ $(n=1,2, \cdots)$ are defined. In case $\mathfrak{X}$ is a Banach space under a weak neighborhood topology $V_{\Gamma}$, it is evident that a necessary and sufficient condition for $X(e)$ to be completely additive relative to the topology $V_{\Gamma}$ is that the real function $\bar{x}(X(e))$ be completely additive for every $\bar{x} \in \Gamma$. In case $\Gamma=\bar{X}$ (or if $\Gamma$ is a determining manifold $\left.\left({ }^{6}\right)\right)$, then it can be proved $[12$, p. $282 ; 5$, p. 326] that complete additivity relative to $V_{\Gamma}$ is equivalent to complete additivity relative to the norm topology. In this case we simply speak of $X(e)$ as com pletely additive.

A real- or infinite-valued function, which is defined on all of $\mathcal{F}$ and is completely additive, is called a measure function over $\mathcal{F}$, and $m(e)$ is the measure of $e$. A measure function $m(e)$ over $\mathcal{F}$ will be assumed given throughout the discussion. A function $X(e)$, defined on a subset of $\mathcal{F}$ to $\mathfrak{X}$, is said to be absolutely continuous provided $m(e)=0$ implies $X(e)=\theta[17$, p. 30]. If $X(e)$ is completely additive on $\mathcal{F}$, then it can be shown $[16$, p. 501] that absolute continuity is equivalent to the property that $\lim m\left(e_{n}\right)=0$ implies $\lim X\left(e_{n}\right)$ $=\theta$. The function $X(e)$ is said to be singular provided there exists $e_{0}$ such that $m\left(e_{0}\right)=0$ and $X(e)=X\left(e \cap e_{0}\right)$, for every $e[17$, p. 30].

3. Integration. A multi-valued function defined on a subset of $\mathcal{F}$ to $\mathfrak{X}$ is said to be contractive provided $e \subseteq e^{\prime}$ implies $x(e) \subseteq x\left(e^{\prime}\right)$, whenever $x(e)$ and $x\left(e^{\prime}\right)$ are defined. Let $\mathfrak{X}(\mathfrak{F})$ denote the class of all such contractive functions defined for elements of $\mathcal{F}$ having finite, nonzero measure. The notation $x(:)$ will be used to indicate a function considered as an element of the class $\mathfrak{X}(\mathfrak{F})$, while $x(e)$ will denote the set of values assumed by the function for the element $e$.

Let $\Delta=\left\{e_{i}\right\}$ be a subdivision of $M$; then the sequence of sets $\left\{x\left(e \cap e_{i}\right) m\left(e \cap e_{i}\right)\right\}$ is denoted by the symbol $I(x, e, \Delta)$. Since $x(e)$ is not defined when $m(e)$ is infinite or zero, it will always be assumed that $m\left(e \cap e_{i}\right)$ is finite and that $x\left(e \cap e_{i}\right) m\left(e \cap e_{i}\right)$ stands for the element $\theta$ whenever $m\left(e \cap e_{i}\right)=0$.

3.1. Definition. A function $x(:) \in \mathfrak{X}(\mathcal{F})$ is said to be $U$-integrable on $e$ provided there exists $X(e) \in \mathbb{X}$ such that if $V \in \mathcal{U}$, then there is a subdivision $\Delta_{V}$ of $e$ such that $I\left(x, e, \Delta_{V}\right)$ is unconditionally summable to $X(e)$ with respect to $V$. $X(e)$ is the U-integral of $x(:)$ on $e$ and we write $X(e)=\int_{x} x(s) d m(s)$.

Except for our admission of a countably additive Boolean algebra instead

(6) $\Gamma$ is a determining manifold $[5$, p. 316] provided that l.u.b. $x \in \Gamma|\bar{x}(x)| /\|\bar{x}\|=\|x\|$. 
of a $\sigma$-field of sets, Definition 3.1 is essentially the same as one given by Garrett Birkhoff [3, p. 51] and exhaustively studied by R. S. Phillips [13]. In the case where $\mathfrak{X}$ is a Banach space under its weak neighborhood topology $V$ and $x(:)$ arises from a point function, Phillips showed this definition to be equivalent to the Pettis definition.

The $U$-integral is a unique, single-valued function of $e$, which is absolutely continuous and completely additive relative to $U$. Also, the integral is linear in $x(:)$; that is, if $x(:), x^{\prime}(:)$ are $U$-integrable on $e$ and $r, r^{\prime}$ are real, then $r x(:)+r^{\prime} x^{\prime}(:)$ is $\mathcal{U}$-integrable on $e$ and

$$
\int_{0}\left(r x(s)+r^{\prime} x^{\prime}(s)\right) d m(s)=r \int_{e} x(s) d m(s)+r^{\prime} \int_{e} x^{\prime}(s) d m(s) .
$$

The proofs of these remarks, as well as that of the next theorem, will be omitted, since they parallel exactly proofs given by Phillips.

3.2 Theorem. If $x(:)$ is $U$-integrable on every $e \subseteq e_{0}$, then, for every $V \in \mathcal{V}$, there exists $\Delta_{V}$ such that, if $\Delta \geqq \Delta_{V}$, then $I(x, e, \Delta)$ is unconditionally summable to $\int_{0} x(s) d m(s)$ with respect to $V$ uniformly for $e \subseteq e_{0}$.

3.3 THEOREM. Let $\left\{e^{n}\right\}$ be a sequence of mutually disjoint elements of $\mathcal{F}$ and, for each $n$, let $x(:)$ be $U$-integrable on every $e \subseteq e^{n}$. If $e^{0}=\sum e^{n}$ and the series

$$
\sum_{n=1}^{\infty} \int_{e \cap e^{n}} x(s) d m(s)
$$

converges unconditionally, uniformly for $e \in \mathcal{F}$, then $x(:)$ is $U$-integrable on every $e \subseteq e^{0}$.

Consider the function

$$
X(e)=\sum_{n=1}^{\infty} \int_{e \cap e^{n}} x(s) d m(s),
$$

which is defined for all $e$. We prove that, for every $e \subseteq e^{0}, x(:)$ is $U$-integrable on $e$ to the value $X(e)$.

Let $V \in \mathcal{U}$ be arbitrary; then, by Theorem 3.2, there exists a subdivision $\Delta_{V}^{n}$ of $e^{n}$ such that if $\Delta \geqq \Delta_{V}^{n}$, then $I\left(x, e \cap e^{n}, \Delta\right)$ is unconditionally summable to $\int_{e} \cap_{e^{n}} x(s) d m(s)$ with respect to $2^{-n-1} V$ uniformly for $e \in \mathcal{F}$. Let $\Delta_{V}$ denote the subdivision of $e^{0}$ which coincides on $e^{n}$ with $\Delta_{V}^{n}$. Then, if $\left\{e_{i}\right\}=\Delta \geqq \Delta_{V}$, there exists $\pi_{\Delta}^{n}$, which is independent of $e$, such that $\sum_{\pi_{V}^{n}} e_{i} \subseteq e^{n}$ and $\pi \supseteq \pi_{\Delta}^{n}$ implies

$$
\int_{e \cap e^{n}} x(s) d m(s)-\sum_{\pi} x\left(e \cap e^{n} \cap e_{i}\right) m\left(e \cap e^{n} \cap e_{i}\right) \subset \frac{1}{2^{n+1}} V,
$$

for every $e$.

Since the series which defines $X(e)$ converges unconditionally, uniformly 
for $e \in \mathcal{F}$, there exists a finite set of positive integers $\nu_{0}$, which is independent of $e$, such that $\nu \supseteq \nu_{0}$ implies

$$
\sum_{\nu} \int_{e \cap e^{n}} x(s) d m(s)-X(e) \in V,
$$

and $\nu^{\prime} \cap \nu_{0}=0$ implies

$$
\pm \sum_{\nu^{\prime}} \int_{e \cap e^{n}} x(s) d m(s) \in V
$$

for every $e$.

Let $\pi_{\Delta}$ denote the union of the sets $\pi_{\Delta}^{n}$, for $n \in \nu_{0}$, and consider $\pi \supseteq \pi_{\Delta}$. The

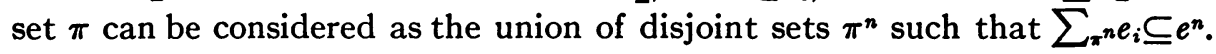
Evidently $\pi^{n} \supseteq \pi_{\Delta}^{n}$, for $n \in \nu_{0}$. Therefore, replacing $\pi$ by $\pi^{n}$ in (1) and summing over $n \in \nu_{0}$, we obtain

$$
\sum_{\nu_{0}} \int_{e \cap e^{n}} x(s) d m(s)-\sum_{x^{\prime}} x\left(e \cap e_{i}\right) m\left(e \cap e_{i}\right) \subset V,
$$

where $\pi^{\prime}$ is the union of the sets $\pi^{n}$, for $n \in \nu_{0}$. It follows from (2) and (4) that

$$
X(e)-\sum_{\pi^{\prime}} x\left(e \cap e_{i}\right) m\left(e \cap e_{i}\right) \subseteq 2 V .
$$

Now let $\pi^{\prime \prime}$ be the union of those sets $\pi^{n}$ for which $n \in \nu_{0}$, and let $\nu^{\prime}$ be a finite set, disjoint from $\nu_{0}$, such that

$$
e_{x^{\prime \prime}}=\sum_{\pi^{\prime \prime}} e_{i} \subseteq \sum_{\prime^{\prime}} e^{n}
$$

Again in (1), replace $e$ by $e \bigcap e_{\pi^{\prime \prime}}$ and sum over $n \in \nu^{\prime}$ to obtain

$$
\sum_{\nu^{\prime}} \int_{e \cap e_{\boldsymbol{x}^{\prime \prime} \cap} \cap e^{n}} x(s) d m(s)-\sum_{\boldsymbol{x}^{\prime \prime}} x\left(e \cap e_{i}\right) m\left(e \cap e_{i}\right) \subset V .
$$

Combining (3) and (6), we have

$$
-\sum_{x^{\prime \prime}} x\left(e \cap e_{i}\right) m\left(e \cap e_{i}\right) \subset 2 V .
$$

This result plus (5) gives

$$
X(e)-\sum_{x} x\left(e \cap e_{i}\right) m\left(e \cap e_{i}\right) \subset 4 V,
$$

and completes the proof.

3.4 Definition $\left({ }^{7}\right)$. A sequence $\left\{x_{n}(:)\right\}$ of elements of $\mathfrak{X}(\mathcal{F})$ is said to converge

(7) That approximate convergence in the ordinary sense is a special case of this is proved in $[16$, Theorems $12.4,15.2]$. See also Theorem 5.2 below. 
approximately to $x(:) \in \mathfrak{X}(\mathcal{F})$ on $e_{0}$ provided, for every $n$ and $V \in U$, there exists a subdivision $\Delta_{n V}$ of $e_{0}$ and $e(n, V) \in \mathcal{F}$ such that $\lim _{n \rightarrow \infty} m(e(n, V))=0$, for every $V$, and $\Delta \geqq \Delta_{n V}$ implies that $I\left(x_{n}, e, \Delta\right), I(x, e, \Delta)$ are summably equal within $V$, for every $e \subseteq e_{0} \cap C e(n, V)$.

The proof of the next theorem will be omitted, since it is contained in the proof of Theorem 9.5 of [16].

3.5 ThEOREM. Let $x_{n}(:)$ be U-integrable on each $e \subseteq e_{0}$ and let $\left\{x^{n}(:)\right\}$ converge approximately to $x(:)$ on $e_{0}$. If

$$
\lim _{n \rightarrow \infty} \int_{e} x_{n}(s) d m(s)
$$

exists for each $e \subseteq e_{0}$, then $x(:)$ is also $U$-integrable on each $e \subseteq e_{0}$ and

uniformly for $e \subseteq e_{0}$.

$$
\lim _{n \rightarrow \infty} \int_{e} x_{n}(s) d m(s)=\int_{e} x(s) d m(s)
$$

4. The Radon-Nikodym theorem. The next theorem plays a fundamental role throughout this section.

4.1 THEOREM. Let $R(e)$ be a real function which is completely additive and defined for all $e \subseteq M^{\prime}$. Let $\mathcal{A}$ denote an arbitrary subclass of $\mathcal{F}$, each of whose elements is contained in $M^{\prime}$. Then there exists a countable (possibly finite), disjoint sequence of elements $e_{n} \in \mathcal{A}$ such that $e \in \mathcal{A}$ and $e \subseteq C\left(\sum e_{n}\right)$ imply $R(e)=0$.

Since $R(e)$ is completely additive, it is evident that, for a given real number $r>0$, there exists at most a finite number of mutually disjoint elements $e \subseteq M^{\prime}$ such that $R(e) \geqq r$. The theorem is an easy consequence of this fact.

4.2 Corollary. If the class $A$ of the theorem contains the join of every disjoint sequence of its elements, then there exists $e(\mathcal{A}) \in \mathcal{A}$ such that $e \in \mathcal{A}$ and $e \subseteq C e(\mathcal{A})$ imply $R(e)=0$.

4.3 TheOREM $\left({ }^{8}\right)$. Let $R(e)$ be a real function, which is completely additive and defined for all $e \subseteq M^{\prime}$. Then $M^{\prime}$ can be decomposed into two disjoint elements $M^{+}, M^{-}$such that $R(e)$ is non-negative for $e \subseteq M^{+}$and is nonpositive for $e \subseteq M^{-}$.

Define the class

$$
A^{+}=\left\{e \mid R\left(e^{\prime}\right) \geqq 0, \text { for } e^{\prime} \subseteq e\right\},
$$

and observe that $A^{+}$is countably additive. Let $M^{+}$denote the element $e\left(\mathcal{A}^{+}\right)$of $\mathcal{A}^{+}$given by Corollary 4.2, and define $M^{-}=M^{\prime} \cap C M^{+}$. It remains to show that $R(e) \leqq 0$, for $e \subseteq M^{-}$.

(8) This theorem is well known for set functions. The proof usually given $[17$, p. 32] will also apply in this case. However we give an independent proof to illustrate use of Corollary 4.2. 
Consider an arbitrary $e^{\prime} \subseteq M^{-}$and define the class

$$
\mathcal{A}^{-}=\left\{e \mid e \subseteq e^{\prime}, R(e) \leqq 0\right\} .
$$

Observe that $\mathcal{A}^{-}$contains the join of any countable disjoint sequence of its elements. Let $e^{-}$be the element $e\left(\mathcal{A}^{-}\right)$of $\mathcal{A}^{-}$given by Corollary 4.2. Then $e \subseteq e^{\prime} \cap C e^{-}$implies $R(e) \geqq 0$; that is, $e^{\prime} \cap C e^{-} \in \mathcal{A}^{+}$. But, since $e^{\prime} \cap C e^{-} \subseteq C M^{+}$, it follows that $R\left(e^{\prime} \cap C e^{-}\right)=0$. Therefore $R\left(e^{\prime}\right) \leqq 0$, and this completes the proof.

4.4 THEOREM. Let $R(e)$ be a real function, which is completely additive and defined for all $e \subseteq M^{\prime}$, where $M^{\prime}$ is of finite measure. Then $M^{\prime}$ can be decomposed into disjoint elements $e^{0}, e^{+}, e^{-}$such that $e \subseteq e^{0}$ implies $|R(e)| \leqq m(e), e \subseteq e^{+} i m$ plies $R(e) \geqq m(e)$, and $e \subseteq e^{-}$implies $R(e) \leqq-m(e)$.

An application of Theorem 4.3 to the function $R(e)-m(e)$, which is obviously completely additive and defined for $e \subseteq M^{\prime}$, gives a decomposition of $M^{\prime}$ into disjoint elements $M_{1}^{+}, M_{1}^{-}$such that $R(e) \geqq m(e)$, for $e \subseteq M_{1}^{+}$, and $R(e) \leqq m(e)$, for $e \subseteq M_{\overline{1}}^{-}$. Similarly, an application of Theorem 4.3 to $R(e)+m(e)$ gives a decomposition of $M^{\prime}$ into disjoint elements $M_{2}^{+}, M_{2}^{-}$such that $R(e) \geqq-m(e)$, for $e \subseteq M_{2}^{+}$, and $R(e) \leqq-m(e)$, for $e \subseteq M_{2}^{-}$. It is evident that a decomposition of the proper kind is given by $e^{0}=M_{1}^{-} \cap M_{2}^{+}, e^{+}=M_{1}^{+}$and $e^{-}=M_{2}^{-} \cap C M_{1}^{+}$.

4.5 THEOREM. Let $R(e)$ be a real function which is completely additive and defined for all $e \subseteq M^{\prime}$, where $M^{\prime}$ is of finite measure. Then there exists a subdivision $\left\{e_{n}\right\}(n=0,1,2, \cdots)$ of $M^{\prime}$ such that, for each $n$ and $e, e^{\prime} \subseteq e_{n}$, it is true that

$$
\left|R(e) m\left(e^{\prime}\right)-R\left(e^{\prime}\right) m(e)\right| \leqq m(e) m\left(e^{\prime}\right) .
$$

In view of Theorem 4.3 , there is no loss in assuming $R(e)$ non-negative for $e \subseteq M^{\prime}$.

Consider first the class $\mathcal{A}$ of all $e \subseteq M^{\prime}$ such that there exists a real number $r(e)$ for which $e^{\prime} \subseteq e$ implies

$$
\left|R\left(e^{\prime}\right)-r(e) m\left(e^{\prime}\right)\right| \leqq m(e) / 2 \text {. }
$$

By application of Theorem 4.1, we obtain a disjoint sequence $\left\{e_{n}\right\}$ $(n=1,2, \cdots)$ of elements of $\mathcal{A}$ such that $e \in \mathcal{A}$ and $e \subseteq C\left(\sum e_{n}\right)$ imply $m(e)=0$. Observe that, for $n \geqq 1$ and $e, e^{\prime} \subseteq e_{n}$,

$$
\begin{aligned}
\left|R(e) m\left(e^{\prime}\right)-R\left(e^{\prime}\right) m(e)\right| \leqq & \left|R(e)-r\left(e_{n}\right) m(e)\right| m\left(e^{\prime}\right) \\
& +\left|r\left(e_{n}\right) m\left(e^{\prime}\right)-R\left(e^{\prime}\right)\right| m(e) \\
\leqq & m(e) m\left(e^{\prime}\right) / 2+m\left(e^{\prime}\right) m(e) / 2=m(e) m\left(e^{\prime}\right) .
\end{aligned}
$$

Therefore the theorem will be proved if we show that (1) is true for $e, e^{\prime} \subseteq e_{0}$, where 


$$
e_{0}=M^{\prime} \cap C\left(\sum e_{n}\right) \text {. }
$$

It will certainly be sufficient to show that $m\left(e_{0}\right)=0$. In order to do this we prove inductively that

$$
2 R(e) \geqq k m(e),
$$

for arbitrary $k$ and $e \subseteq e_{0}$. Since $R\left(e_{0}\right)$ is finite, it will follow immediately that $m\left(e_{0}\right)=0$.

The function $R(e)$ is assumed non-negative for $e \subseteq M^{\prime}$; therefore (2) holds for $k=0$. Hence assume it true for $k=0,1,2, \cdots, n$. Then the function

$$
R_{n}(e)=2 R(e)-n m(e)
$$

is non-negative, completely additive and defined for $e \subseteq e_{0}$. Theorem 4.4 can thus be applied to obtain disjoint elements $e^{0}, e^{+}$such that $e_{0}=e^{0} \cup e^{+}, e \subseteq e^{0}$ implies $\left|R_{n}(e)\right| \leqq m(e)$, and $e \subseteq e^{+}$implies $R_{n}(e) \geqq m(e)$. But $e^{0} \in \mathcal{A}$, therefore $m\left(e^{0}\right)=0$. It follows that $m\left(e_{0}\right)=m\left(e^{+}\right)$and, hence, that $R_{n}(e) \geqq m(e)$, for $e \subseteq e_{0}$. In other words, $2 R(e) \geqq(n+1) m(e)$, for $e \subseteq e_{0}$. This completes the proof that (1) holds for all $k$ and also completes the proof of the theorem.

We now assume that $\mathfrak{X}$ is an arbitrary Banach space topologized by the weak neighborhood system $V_{\Gamma}$, where $\Gamma$ is a total subset of $\bar{x}$.

4.6 THEOREM. If $M$ is the join of a countable number of elements of finite measure and $X(e)$ is any function, with values in $\mathfrak{X}$ and defined for elements of $\mathfrak{F}$ having finite measure, such that $\bar{x}(X(e))$ is completely additive for every $\bar{x} \in \Gamma$, then a necessary and sufficient condition that $X(e)$ be representable as a $V_{\Gamma^{-}}$ integral is that $X(e)$ be absolutely continuous.

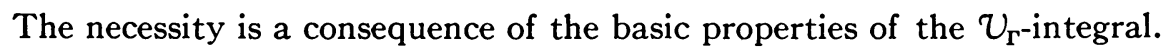
To prove the sufficiency, we shall show that $X(e)$ is the $V_{r}$-integral of the function

$$
x(e)=\left\{X\left(e^{\prime}\right) / m\left(e^{\prime}\right) \mid e^{\prime} \subseteq e, m\left(e^{\prime}\right) \neq 0\right\},
$$

on every $e$ of finite measure. It is obvious that $x(:) \in \mathfrak{X}(\mathcal{f})$.

Let $M^{k}(k=1,2, \cdots)$ be mutually disjoint elements of finite measure such that $\sum M^{k}=M$. Let $V$ be an arbitrary element of $V_{\Gamma}$ defined by $\epsilon>0$ and $\bar{x}_{i} \in \Gamma(i=1,2, \cdots, v)$. Consider the real functions

$$
R_{i}^{k}(e)=\epsilon^{-1} 2^{k+2} m\left(M^{k}\right) \bar{x}_{i}(X(e)) \quad(i=1,2, \cdots, v ; k=1,2, \cdots) .
$$

Evidently $R_{\mathfrak{l}}^{k}(e)$ is completely additive and defined for $e \subseteq M^{k}$. In Theorem 4.5, for a particular choice of $k$ and $i$, take $R(e)=R_{i}^{k}(e), M^{\prime}=M^{k}$ and denote by $\Delta_{l}^{k}$ the subdivision of $M^{k}$ given by the theorem. Then let $\Delta^{k}$ be the subdivision of $M^{k}$ obtained by taking the product of the subdivisions $\Delta_{i}^{k}$ for $i=1,2, \cdots, v$. Finally, let $\Delta_{V}=\left\{e_{n}\right\}$ be the subdivision of $M$ which coincides with $\Delta^{k}$ on $M^{k}$. 
From the manner in which the subdivision $\Delta_{V}$ was defined, it is clear that if $e_{n} \subseteq M^{k}$ and $e^{\prime}, e^{\prime \prime} \subseteq e_{n}$, then

$$
\begin{array}{r}
\left|\bar{x}_{i}\left(X\left(e^{\prime}\right)\right) m\left(e^{\prime \prime}\right)-\bar{x}_{i}\left(X\left(e^{\prime \prime}\right)\right) m\left(e^{\prime}\right)\right| \leqq m\left(e^{\prime}\right) m\left(e^{\prime \prime}\right) \epsilon / 2^{k+2} m\left(M^{k}\right) \\
(i=1,2, \cdots, v) .
\end{array}
$$

Since $X(e)$ is absolutely continuous $\left({ }^{9}\right)$, it follows from (3) that $\left({ }^{10}\right)$, for every $e \in \mathcal{F}$ and $e_{n} \subseteq M^{k}$,

$$
\begin{array}{r}
\left|\bar{x}_{i}\left(X\left(e \cap e_{n}\right)\right)-\bar{x}_{i}\left(x\left(e \cap e_{n}\right)\right) m\left(e \cap e_{n}\right)\right| \leqq m\left(e \cap e_{n}\right) \epsilon / 2^{k+2} m\left(M^{k}\right) \\
(i=1,2, \cdots, v) .
\end{array}
$$

Summation of (4) over $n \in \pi$ yields

$$
\begin{aligned}
\left|\bar{x}_{i}\left(\sum_{\pi} X\left(e \cap e_{n}\right)\right)-\bar{x}_{i}\left(\sum_{\pi} x\left(e \cap e_{n}\right) m\left(e \cap e_{n}\right)\right)\right| & \leqq \epsilon / 2 \\
(i & =1,2, \cdots, v) .
\end{aligned}
$$

If $e$ is of finite measure, then, since $\bar{x}_{i}(X(e))$ is completely additive, there exists $\pi_{V}$ such that $\pi \supseteq \pi_{V}$ implies

$$
\left|\bar{x}_{i}(X(e))-\bar{x}_{i}\left(\sum_{x} X\left(e \cap e_{n}\right)\right)\right|<\epsilon / 2 \quad(i=1,2, \cdots, v) .
$$

Therefore, combining (5) and (6), we obtain

$$
X(e)-\sum_{x} x\left(e \cap e_{n}\right) m\left(e \cap e_{n}\right) \subset V, \text { for } \pi \supseteq \pi_{V} .
$$

In other words, the sequence of sets $I\left(x, e, \Delta_{V}\right)$ is unconditionally summable to $X(e)$ with respect to $V$. Since $V$ is arbitrary, we have proved $X(e)$ $=\int_{e} x(s) d m(s)$.

In case $\left({ }^{11}\right) \Gamma=\bar{X}$, the condition that $\bar{x}(X(e))$ be completely additive, for every $\bar{x} \in \Gamma$, is equivalent to the condition that $X(e)$ be completely additive (in the norm topology). In this case Theorem 4.6 has the following form.

4.7 THEOREM. If $M$ is the join of a countable number of elements of finite measure and $X(e)$ is any completely additive function with values in $\mathfrak{X}$ and defined for elements of $\mathcal{F}$ having finite measure, then a necessary and sufficient

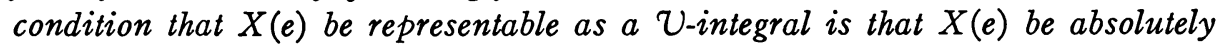
continuous.

$\left(^{9}\right)$ Observe that (3) is obtained without the assumption of absolute continuity. However, since we have made the convention that $x(e) m(e)$ stand for $\theta$ when $m(e)=0$, absolute continuity is needed in order for (4) to hold when $m\left(e \cap e_{n}\right)=0$.

(10) The notation $\bar{x}(x(c))$ stands for the set of real numbers $\{\bar{x}(x) \mid x \in x(e)\}$. Also, if $A$ is a set of real numbers and $k$ is a constant, then $|A| \leqq k$ means that $|r| \leqq k$, for every $r \in A$.

(11) Or if $\Gamma$ is a determining manifold. 
It is not difficult to prove the following generalization of the Lebesgue decomposition theorem $\left({ }^{12}\right)[17$, p. 35].

4.8 THEOREM. Every completely additive function $X(e)$, with values in $\mathfrak{X}$ and defined for elements of $\mathcal{Z}$ having finite measure, can be represented uniquely as the sum of an absolutely continuous and a singular part, both of which are also completely additive.

Theorems 4.7 and 4.8 give immediately the next theorem.

4.9 THEOREM. If $M$ is the union of a countable number of elements of finite measure, then any completely additive function $X(e)$, with values in $\mathfrak{X}$ and defined for elements of $\mathcal{F}$ having finite measure, can be represented in the form $\left({ }^{18}\right)$

$$
X(e)=X_{0}(e)+\int_{e} x(s) d m(s),
$$

where $X_{0}(e)$ is completely additive and singular and the integral is the U-integral.

5. Representation of linear transformations on summable functions to a Banach space. In this section, $\mathcal{F}$ is taken to be a $\sigma$-field of subsets of an abstract set $M$. It will be assumed throughout that $M$ is the union of a countable number of mutually disjoint sets $M^{k}$ of finite measure. The Banach space of real-valued, summable point functions defined over $M$ is denoted by $L(M)$. The point function $f(p)$, considered as an element of $L(M)$, will be denoted by the symbol $f($.$) . Associated with f($.$) is the contractive func-$ tion $f(:)$ defined by

$$
f(e)=\{f(p) \mid p \in e, e \in \mathcal{F}\} .
$$

The integral defined in $\$ 3$ reduces in this case to the ordinary Lebesgue integral, which we write as $\int_{e} f(s) d m(s)$ to conform with the notation of $\S 3$. Thus, the norm of $f(.) \in L(M)$ is

$$
\|f(.)\|=\int_{M}|f(s)| d m(s) .
$$

If $x(:) \in \mathfrak{X}(\mathcal{F})$ and $f(.) \in L(M)$, the function $x(:) f(:)$ is defined by

$$
x(e) f(e)=\{x r \mid x \in x(e), r \in f(e)\} .
$$

It is evident that $x(:) f(:) \in \mathfrak{X}(\mathcal{F})$. The object of this section is to obtain a $U$-integral representation for the general bounded linear transformation on $L(M)$ to an arbitrary Banach.

(12) The proof involves an application of Corollary 4.2 in which $\mathcal{A}$ consists of all elements of $\mathcal{F}$ which are of measure zero. See [15, Theorem 4.5 ].

(13) This decomposition is unique to the extent given by Theorem 4.7 ; however the function $x(:)$ is not unique. 
5.1 Lemma. Let $V \in \mathcal{U}$ and let $x_{i}(i=1,2, \cdots, n)$ be any finite set of elements of $\mathfrak{X}$ such that $\sum_{\pi} x_{i} \in V$, for every subset $\pi$ of the integers $(1,2, \cdots, n)$. Then, for arbitrary real numbers $r_{i}$ such that $\left|r_{i}\right| \leqq r$, it is true that $\sum_{i=1}^{n} r_{i} x_{i} \in 2 r V$.

Assume first that $0 \leqq r_{i} \leqq r, r_{i-1} \leqq r_{i}$ and define $y_{i}=\sum_{j=1}^{n} x_{j}, c_{i}=\left(r_{i}-r_{i-1}\right) r^{-1}$, with $c_{1}=r_{1}$. Observe that $c_{i} \geqq 0$ and $\sum c_{i} \leqq 1$. Since $V$ is a convex set and contains $\theta$ as well as each of the elements $y_{i}$, it follows immediately that $\sum c_{i} y_{i} \in V$. But observe that $\sum c_{i} y_{i}=r^{-1} \sum r_{i} x_{i}$; therefore $\sum r_{i} x_{i} \in r V$. Similarly, if $-r \leqq r_{i} \leqq 0$, we can obtain $\sum r_{i} x_{i} \in-r V$. Now let $\left|r_{i}\right| \leqq r$ be arbitrary and define

$$
\begin{aligned}
& r_{i}^{+}= \begin{cases}r_{i}, & \text { if } r_{i} \geqq 0, \\
0, & \text { if } r_{i}<0,\end{cases} \\
& r_{i}^{-}= \begin{cases}r_{i}, & \text { if } r_{i}<0, \\
0, & \text { if } r_{i} \geqq 0 .\end{cases}
\end{aligned}
$$

Then $\sum r_{i}^{+} x \in r V$ and $\sum r_{i}^{-} x \in-r V$. Hence

$$
\sum r_{i} x_{i}=\sum r_{i}^{+} x_{i}+\sum r_{i}^{-} x_{i} \in r V-r V \subseteq 2 r V,
$$

which completes the proof.

5.2 THEOREM. If $f_{n}($.$) converges approximately to f($.$) in the ordinary sense$ and $x(:)$ is $U$-integrable on every $e \subseteq e_{0}$, then $x(:) f_{n}(:)$ converges approximately to $x(:) f(:)$ on $e_{0}$ in the sense of Definition 3.4.

For every $n$ and $\delta>0$, there exists $e(n, \delta) \in \mathcal{F}$ such that, for each $\delta$, $\lim _{n \rightarrow \infty} m(e(n, \delta))=0$ and

$$
\left|f_{n}(p)-f(p)\right|<\delta, \quad p \in C e(n, \delta) .
$$

This implies

$$
f_{n}(e) \subseteq f(e)+I_{\delta}, \quad f(e) \subseteq f_{n}(e)+I_{\delta},
$$

where $I_{\delta}$ is the interval $(-\delta, \delta)$ and $e \subseteq C e(n, \delta)$.

Let $V \in U$ be arbitrary. Since $x(:)$ is $U$-integrable on every $e \subseteq e_{0}$, there exists, by Theorem 3.2, a subdivision $\Delta_{V}$ of $e_{0}$ such that, if $\Delta \geqq \Delta_{V}$, then $I(x, e, \Delta)$ is unconditionally summable to $\int_{e} x(s) d m(s)$ with respect to $V$ uniformly for $e \subseteq e_{0}$. That is, if $\Delta=\left\{e_{i}\right\}$, there exists $\pi_{\Delta}$, which is independent of $e$, such that $\pi \supseteq \pi_{\Delta}$ implies

$$
\sum_{\pi} x\left(e \cap e_{i}\right) m\left(e \cap e_{i}\right) \subset \int_{0} x(s) d m(s)+V,
$$

for every $e \subseteq e_{0}$. Since the $U$-integral is completely additive, $\int_{\theta} x(s) d m(s)$ is bounded for $e \subseteq e_{0}$ [15, Corollary 2.5]; therefore, there exists $k_{V}$ which is independent of $e \subseteq e_{0}$, such that 


$$
\sum_{\pi} x\left(e \cap e_{i}\right) m\left(e \cap e_{i}\right) \subset k_{V} V,
$$

for $\pi \supseteq \pi_{\Delta}$ and $e \subseteq e_{0}$. Since (2) holds for arbitrary $e \subseteq e_{0}$, the restriction that $\pi$ contain $\pi_{\Delta}$ can also be dropped. From (2) and Lemma 5.1 it follows that

$$
\sum_{x} I_{\delta} x\left(e \cap e_{i}\right) m\left(e \cap e_{i}\right) \subset 2 \delta k_{V} V,
$$

for arbitrary $\pi$ and $\delta>0$.

Now define $\delta_{V}=\left(2 k_{V}\right)^{-1}, e(n, V)=e\left(n, \delta_{V}\right)$ and $F(e)=x(e) f(e) m(e)$. Then, for $e \subseteq e_{0} \cap C e(n, V)$, (1) implies

$$
\begin{aligned}
F_{n}\left(e \cap e_{i}\right) & \subseteq F\left(e \cap e_{i}\right)+I_{\delta_{V}} x\left(e \cap e_{i}\right) m\left(e \cap e_{i}\right), \\
F\left(e \cap e_{i}\right) & \subseteq F_{n}\left(e \cap e_{i}\right)+I_{\delta_{V}} x\left(e \cap e_{i}\right) m\left(e \cap e_{i}\right) .
\end{aligned}
$$

If we sum relations (4) over arbitrary $\pi$ and apply (3), the result is

$$
\begin{aligned}
& \sum_{\pi} F_{n}\left(e \cap e_{i}\right) \subseteq \sum_{x} F\left(e \cap e_{i}\right)+V, \\
& \sum_{\pi} F\left(e \cap e_{i}\right) \subseteq \sum_{x} F_{n}\left(e \cap e_{i}\right)+V .
\end{aligned}
$$

This means that the sequences $\left\{x\left(e \cap e_{i}\right) f_{n}\left(e \cap e_{i}\right) m\left(e \cap e_{i}\right)\right\}$ and $\left\{x\left(e \cap e_{i}\right) f\left(e \cap e_{i}\right) m\left(e \cap e_{i}\right)\right\}$ are summably equal within $V$ for every $e \subseteq e_{0} \cap \operatorname{Ce}(n, V)$. Since $\lim _{n \rightarrow \infty} m(e(n, V))=0$, the proof is complete.

5.3 Definition. Let $x(:)$ be U-integrable on every e of finite measure. Then

$$
\|x(:)\|=\underset{0<m(e)<\infty}{\text { l.u.b. }} \frac{1}{m(e)}\left\|\int_{e} x(s) d m(s)\right\| .
$$

5.4 TheoRem. Let $x(:)$ be $U$-integrable on every e of finite measure. Then a necessary and sufficient condition that $x(:) f(:)$ be U-integrable on e, for arbitrary $f(.) \in L(M)$ and $e \in \mathcal{F}$, is that $\|x(:)\|$ be finite. Moreover,

$$
\left\|\int_{e} x(s) f(s) d m(s)\right\| \leqq\|x(:)\| \int_{e}|f(s)| d m(s),
$$

whenever the integral on the left is defined.

The sufficiency will be proved first. Let $e^{0}$ be any set of finite measure and denote its characteristic function by $c_{e} 0(p)$. It is obvious that $c_{\theta} 0(.) \in L(M)$ and $x(:) c_{\theta^{0}}(:)$ is $U$-integrable on every $e \in \mathcal{F}$. As a matter of fact,

$$
\int_{e} x(s) c_{e^{0}}(s) d m(s)=\int_{e \cap e^{0}} x(s) d m(s)
$$

It follows easily from these remarks that if $f($.$) is a step function which be-$ 
longs to $L(M)$, then $x(:) f(:)$ is $U$-integrable on every $e$ and the inequality (5) holds.

Now let $f($.$) be an arbitrary element of L(M)$. Then there exists a sequence of step functions $f_{n}(.) \in L(M)$ such that

$$
\lim _{n \rightarrow \infty} \int_{e}\left|f_{n}(s)-f(s)\right| d m(s)=0,
$$

The sequence $\left\{f_{n}().\right\}$ will also converge approximately to $f($.$) in the ordinary$ sense. Thus, by Theorem $5.2,\left\{x(:) f_{n}(:)\right\}$ converges approximately to $x(:) f(:)$ in the sense of Definition 3.4 on every set $e_{0}$ of finite measure. Moreover, since $f_{m}(p)-f_{n}(p)$ is a step function,

$$
\left\|\int_{e} x(s) f_{m}(s) d m(s)-\int_{e} x(s) f_{n}(s) d m(s)\right\| \leqq\|x(:)\| \int_{e}\left|f_{n}(s)-f_{n}(s)\right| d m(s) .
$$

Therefore,

$$
\lim _{n \rightarrow \infty} \int_{e} x(s) f_{n}(s) d m(s)
$$

exists for every $e \in \mathcal{F}$. By Theorem 3.5, it follows that $x(:) f(:)$ is $U$-integrable on every $e$ of finite measure and

$$
\left\|\int_{e} x(s) f(s) d m(s)\right\| \leqq\|\bar{x}(:)\| \int_{e}|f(s)| d m(s) .
$$

Now let $e^{0}$ be an arbitrary element of $\mathcal{F}$. Then $e^{0}$ can be expressed as the join of a countable sequence $\left\{e^{n}\right\}$ of mutually disjoint sets of finite measure. Also, since $\int_{\theta}|f(s)| d m(s)$ is defined and completely additive over all of $\mathcal{F}$, (6) implies that the series $\sum \int_{e \cap^{n} n} x(s) f(s) d m(s)$ converges unconditionally, uniformly for $e \in \mathcal{F}$. Hence Theorem 3.3 applies, and we conclude that $x(:) f(:)$ is $U$-integrable on $e^{0}$. That (6) holds for all $e \in \mathcal{F}$ is a consequence of complete additivity.

Assume that $x(:) f(:)$ is $U$-integrable on $e$, for arbitrary $f(.) \in L(M)$ and $\mathrm{e} \in \mathcal{F}$. Let $\bar{x} \in \overline{\mathfrak{X}}$ and define

$$
S(e)=\bar{x}\left(\int_{e} x(s) d m(s)\right) .
$$

The function $S(e)$, which is defined for every $e$ of finite measure, is completely additive and absolutely continuous. It will now be proved that there exists a constant $K(\bar{x})<\infty$ such that

$$
|S(e)| \leqq K(\bar{x}) m(e),
$$

for every $e$ of finite measure. Suppose such $K(\bar{x})$ does not exist, then two cases arise: (i) For each $M^{k}$ there exists $K^{k}(\bar{x})$. such that $e \subseteq M^{k}$ implies 
$|S(e)| \leqq K^{k}(\bar{x}) m(e)$; (ii) There is a $k_{0}$ such that, for every $n$, there exist sets $e$ of finite nonzero measure contained in $M^{k_{0}}$ such that $|S(e)|>n^{2} m(e)$.

In case (i), we obtain immediately a sequence of disjoint sets $e_{n}$ such that $0<m\left(e_{n}\right)<\infty$ and $\left|S\left(e_{n}\right)\right|>n^{2} m\left(e_{n}\right)$. Define the real function

Then

$$
f(p)=\left\{\begin{array}{cl}
n^{-2}\left(m\left(e_{n}\right)\right)^{-1}, & \text { for } p \in e_{n}, \\
0, & \text { for } p \in C\left(\sum e_{n}\right) .
\end{array}\right.
$$

$$
\int_{M}|f(s)| d m(s)=\sum \int_{e_{n}}|f(s)| d m(s)=\sum 1 / n^{2}<\infty ;
$$

hence $f(.) \in L(M)$ and $x(:) f(:)$ is $U$-integrable on every $e$. Observe that

$$
\bar{x}\left(\int_{M} x(s) f(s) d m(s)\right)=\sum \frac{1}{n^{2} m\left(e_{n}\right)} \bar{x}\left(\int_{e_{n}} x(s) d m(s)\right)=\sum \frac{S\left(e_{n}\right)}{n^{2} m\left(e_{n}\right)} .
$$

Since $\left|S\left(e_{n}\right)\right|>n^{2} m\left(e_{n}\right)$, the series cannot converge. This contradiction disposes of case (i).

In case (ii), since the set $M^{k_{0}}$ can be decomposed into two disjoint sets on each of which $S(e)$ is of constant sign, there is no loss in assuming $S(e) \geqq 0$, for $e \subseteq M^{k_{0}}$. We now define inductively a sequence $\left\{s_{n}\right\}$ of subsets of $M^{k_{0}}$ such that $s_{k} \subseteq s_{k-1}$ and

$$
\begin{array}{ll}
S(e) \geqq k^{2} m(e), & e \subseteq s_{k}, \\
S(e) \leqq k^{2} m(e), & e \subseteq M^{k_{0}} \cap C s_{k} .
\end{array}
$$

Let $s_{0}=M^{k_{0}}$ and assume $s_{k}$ defined for $k=0,1,2, \cdots, n-1$ and satisfying the desired properties. In Theorem 4.3 take $R(e)=n^{-2} S(e), M^{\prime}=s_{n-1}$ and denote by $s_{n}$ the set $e^{+}$given by the theorem. Then $e \subseteq s_{n}$ implies $S(e) \geqq n^{2} m(e)$. Since $S(e)$ is assumed non-negative on $M^{k_{0}}$, the set $e^{-}$given by the theorem is of measure zero; therefore, if $e \subseteq s_{n-1} \cap C s_{n}, S(e) \leqq n^{2} m(e)$. Since $e \subseteq M^{k_{0}} \cap C s_{n-1}$ implies $S(e) \leqq(n-1)^{2} m(e) \leqq n^{2} m(e)$, it follows that $S(e) \leqq n^{2} m(e)$, for $e \subseteq M^{k_{0}} \cap C s_{n}$. An induction now gives the desired sequence $\left\{s_{n}\right\}$. Moreover, by the hypothesis of case (ii), $m\left(s_{n}\right)>0$, for every $n$. Also, since $S(e)$ is bounded for $e \subseteq M^{k_{0}}, \lim _{n \rightarrow \infty} m\left(s_{n}\right)=0$. Therefore, there exist $n_{k}$ such that $m\left(s_{n_{k-1}} \cap C s_{n_{k}}\right)>0, k=1,2, \cdots$. The sets $e_{k}=s_{n_{k}} \cap C s_{n_{k-1}}$ are mutually disjoint and $e \subseteq e_{k}$ implies $S(e) \geqq n_{k}^{2} m(e)$. This situation leads to a contradiction, just as in case (i), and completes proof of the existence of $K(\bar{x})$ satisfying (7).

We have proved that

$$
\underset{0<m(e)<\infty}{\text { l.u.b. }}\left|\bar{x}\left(\frac{1}{m(e)} \int_{e} x(s) d m(s)\right)\right|<\infty,
$$

for every $\bar{x} \in \overline{\mathfrak{X}}$. By a theorem proved by $\mathrm{N}$. Dunford [5, Theorem 2], this implies 


$$
\underset{0<m(e)<\infty}{\operatorname{l.u} . b .}\left\|\frac{1}{m(e)} \int_{0} x(s) d m(s)\right\|<\infty,
$$

which completes proof of the theorem.

5.5 Corollary. Let $x(:)$ be U-integrable on every set of finite measure. Then a necessary and sufficient condition that

$$
T f(.)=\int_{M} x(s) f(s) d m(s)
$$

define a bounded linear transformation on $L(M)$ to $\mathfrak{X}$ is that $\|x(:)\|$ be finite. Moreover, $\|x(:)\|=\|T\|$.

The representation theorem which follows is a generalization of Theorem 2.1.6 of [7].

5.6 THEOREM. Every bounded linear transformation $T f($.$) on L(M)$ to $\mathfrak{X}$ has a $U$-integral representation of the form

$$
T f(.)=\int_{M} x(s) f(s) d m(s),
$$

where $x(:)$ is U-integrable on every set of finite measure and $\|T\|=\|x(:)\|$.

Let $e$ be of finite measure and consider the set function

$$
X(e)=T c_{e}(.),
$$

where $c_{\theta}($.$) is the characteristic function of e$. The function $X(e)$, which is defined for every $e$ of finite measure, is completely additive and absolutely continuous. Hence, by Theorem 4.6, there exists $x(:) \in \mathfrak{X}(\mathcal{F})$, integrable on sets of finite measure, such that

$$
X(e)=\int_{e} x(s) d m(s) .
$$

Observe that

$$
\|X(e)\| \leqq\|T\| m(e),
$$

for $e$ of finite measure; hence $\|x(:)\|$ is finite and

$$
T^{\prime} f(.)=\int_{M} x(s) f(s) d m(s)
$$

defines a bounded linear transformation on $L(M)$ to $\mathfrak{X}$ with $\|x(:)\|=\left\|T^{\prime}\right\|$. Observe that, if $f(.) \in L(M)$ is a step function, then

$$
T f(.)=T^{\prime} f(.) \text {. }
$$


Since step functions are dense in $L(M)$, it follows that $T f($.$) and T^{\prime} f($.$) are$ identical.

\section{BIBLIOGRAPHY}

1. S. Banach, Theorie des operations linéaires, Monografje Matematyczme, Warsaw, 1934.

2. Garrett Birkhoff, Lattice theory, Amer. Math. Soc. Colloquium Publications, vol. 25, New York, 1940. 39-56.

3. - Moore-Smith convergence in general topology, Ann. of Math. vol. 38 (1937) pp.

4. James A. Claikson, Uniformly convex spaces, Trans. Amer. Math. Soc. vol. 40 (1936) pp. $396-414$.

5. N. Dunford, Uniformity in linear spaces, Trans. Amer. Math. Soc. vol. 44 (1938) pp. $305-356$.

6. N. Dunford and A. P. Morse, Remarks on the preceding paper of James A. Clarkson, Trans. Amer. Math. Soc. vol. 40 (1936) pp. 415-420.

7. N. Dunford and B. J. Pettis, Linear operations on summable functions, Trans. Amer. Math. Soc. vol. 47 (1940) pp. 323-392.

8. I. Gelfand, Abstrakte Funktionen und lineare Operatoren, Rec. Math. (Mat. Sbornik) N.S. vol. 4 (1938) pp. 235-284.

9. T. H. Hildbrandt, On unconditional convergence in normed vector spaces, Bull. Amer. Math. Soc. vol. 46 (1940) pp. 957-962. pp. 1-20.

10. J. von Neumann, On complete topological spaces, Trans. Amer. Math. Soc. vol. 37 (1935)

11. B. J. Pettis, Differentiation in Banach spaces, Duke Math. J. vol. 5 (1939) pp. 254-269. 304.

12. - On integration in vector spaces, Trans. Amer. Math. Soc. vol. 44 (1938) pp. 277-

13. R. S. Phillips, Integration in a convex linear topological space, Trans. Amer. Math. Soc. vol. 47 (1940) pp. 114-145.

14. - On weakly compact subsets of a Banach space, Amer. J. Math. vol. 55 (1943) pp. 108-136.

15. C. E. Rickart, Decomposition of additive set functions, Duke Math. J. vol. 9 (1943) pp. 653-665.

16. - Integration in a convex linear topological space, Trans. Amer. Math. Soc. vol. 52 (1942) pp. 498-521.

17. S. Saks, Theory of the integral, Monografje Matematyczme, Warsaw, 1937.

Yale University, New Haven, Conn. 